\title{
A randomized, double-blind, multicenter, placebo-controlled phase III trial to evaluate the efficacy and safety of pregabalin in Japanese patients with fibromyalgia
}

Hiroyoshi Ohta ${ }^{1}$, Hiroshi Oka², Chie Usui ${ }^{3}$, Masayuki Ohkura ${ }^{1}$, Makoto Suzuki ${ }^{1}$ and Kusuki Nishioka ${ }^{4^{*}}$

\begin{abstract}
Introduction: Fibromyalgia is a chronic disorder characterized by widespread pain and tenderness. Prior trials have demonstrated the efficacy of pregabalin for the relief of fibromyalgia symptoms, and it is approved for the treatment of fibromyalgia in the United States. However, prior to this study, there has not been a large-scale efficacy trial in patients with fibromyalgia in Japan.

Methods: This randomized, double-blind, multicenter, placebo-controlled trial was conducted at 44 centers in Japan to assess the efficacy and safety of pregabalin for the symptomatic relief of pain in fibromyalgia patients. Patients aged $\geq 18$ years who had met the criteria for fibromyalgia were randomized to receive either pregabalin, starting at $150 \mathrm{mg} /$ day and increasing to a maintenance dose of 300 or $450 \mathrm{mg} / \mathrm{day}$, or placebo, for 15 weeks. The primary efficacy endpoint was mean pain score at final assessment. Secondary endpoints included Patient Global Impression of Change (PGIC) together with measures of sleep, physical functioning and quality of life.
\end{abstract}

Results: A total of 498 patients (89\% female) were randomized to receive either pregabalin $(n=250)$ or placebo $(n=248)$. Pregabalin significantly reduced mean pain score at final assessment (difference in mean change from baseline, compared with placebo $-0.44 ; P=0.0046)$ and at every week during the study $(P<0.025)$. Key secondary endpoints were also significantly improved with pregabalin treatment compared with placebo, including PGIC (percentage reporting symptoms "very much improved" or "much improved", $38.6 \%$ vs $26.7 \%$ with placebo; $P=0.0078$ ); pain visual analog scale (difference in mean change from baseline, compared with placebo $-6.19 ; P=0.0013$ ); Fibromyalgia Impact Questionnaire total score $(-3.33 ; P=0.0144)$; and quality of sleep score $(-0.73 ; P<0.0001)$. Treatment was generally well tolerated, with somnolence and dizziness the most frequently reported adverse events.

Conclusions: This trial demonstrated that pregabalin, at doses of up to $450 \mathrm{mg} /$ day, was effective for the symptomatic relief of pain in Japanese patients with fibromyalgia. Pregabalin also improved measures of sleep and functioning and was well tolerated. These data indicate that pregabalin is an effective treatment option for the relief of pain and sleep problems in Japanese patients with fibromyalgia.

Trial Registration: ClinicalTrials.gov: NCT00830167

\footnotetext{
* Correspondence: imse-kn@tokyo-med.ac.jp

${ }^{4}$ Institute of Innovative Medical Science and Education, Tokyo Medical

University, 6-1-1 Shinjyuku, Shinjyuku-ku, Tokyo 160-8402, Japan

Full list of author information is available at the end of the article
} 


\section{Introduction}

Fibromyalgia (FM) is a common chronic pain disorder characterized by widespread pain and tenderness [1-5]. Associated symptoms include fatigue, sleep disturbance, headache, mood disorders and memory or concentration problems $[1,6,7]$. Of the symptoms of FM, both patients and clinicians have ranked pain as the most important [6]. An estimate of the prevalence of FM in Japan by a Ministry of Health, Labor and Welfare study group was $1.7 \%$, with a higher incidence in women (male-to-female ratio 1:4.8) and a mean age of $51.5 \pm 16.9$ years [8,9]. However, FM remains an underdiagnosed disease and it can be challenging for patients to obtain appropriate treatment. At the time of this study, there was no approved medication for FM in Japan. Pregabalin was approved in Japan for the treatment of pain associated with FM in June 2012.

Previous trials conducted in the United States (US) [10-13] and internationally [14] have demonstrated the safety and efficacy of pregabalin, an $\alpha_{2} \delta$ ligand with analgesic and anticonvulsant activity [15], in the treatment of FM. In these trials, pregabalin (at 300 to $600 \mathrm{mg} /$ day) demonstrated significant improvements in pain, sleep, function and patient impressions of change compared with placebo [10-14]. However, prior to the current study, there has not been a clinical trial conducted in FM patients in Japan.

The objective of this trial was to assess the efficacy and safety of pregabalin (300 or $450 \mathrm{mg} /$ day) compared with placebo in patients with FM in Japan. The primary objective was to assess the effect of pregabalin on the symptomatic relief of pain. Key secondary objectives included evaluation of the safety and tolerability of pregabalin, together with its effect on sleep, physical functioning, patient impressions of change and health-related quality of life.

\section{Materials and methods Study design}

This was a randomized, double-blind, multicenter, placebo-controlled trial to compare the efficacy and safety of pregabalin vs placebo in patients with FM. The study was conducted at 44 study centers in Japan between March 2009 and May 2011. Patients were randomized to receive pregabalin, starting at $150 \mathrm{mg} /$ day and increasing to a maintenance dose of 300 or $450 \mathrm{mg} /$ day, or placebo. The study included a 1-week, single-blind, placebo runin period; a 3-week dose-escalation/optimization phase; a 12-week, fixed-dose treatment period; and a 1-week taper phase (Figure 1).

Patients were registered to the randomization control system (IMPALA), which provided subject randomization numbers. Pregabalin and identical placebo capsules were prescribed by the investigator using blinded drug numbers issued by IMPALA.

This study was conducted in accordance with the International Conference on Harmonization guidelines on Good Clinical Practice. The study protocol and informed consent documents were approved by the relevant Institutional Review Boards and Independent Ethics Committees. Written informed consent was obtained from all patients prior to their inclusion in the study. This study is registered at clinicaltrials.gov under the identifier NCT00830167.

\section{Patient population}

Methodology and screening criteria were similar to those used in previously reported studies of pregabalin $[10,14]$. Patients were aged $\geq 18$ years and had met the 1990 ACR (American College of Rheumatology) criteria for FM [1]. Patients also had a score of $\geq 40 \mathrm{~mm}$ on the $100 \mathrm{~mm}$ pain visual analog scale (VAS) at Visit 2, and had assessed and documented their pain score on at least four of the past seven days prior to Visit 2 while recording an average pain score of $\geq 4$ on the 11-point numeric rating scale. Patients were excluded if they had a decrease of $\geq 30 \%$ on their pain VAS during the placebo run-in period (at Visit 2 compared with Visit 1), in order to remove potential placebo-responders. Patients were also excluded if they were being treated for depression, were at risk of suicide or self-harm in the opinion of the study investigator, had an active malignancy or a history of malignancy, had a creatinine clearance rate $\leq 60 \mathrm{ml} / \mathrm{min}$, or experienced pain which might potentially affect assessment or self-evaluation of FM.

\section{Study medication}

Dosing was twice each day (morning and evening) based on the US prescribing information for pregabalin in FM [16]. The individual investigators decided whether to advise patients to take the study drug before or after meals, and patients were directed to take medication in the same manner throughout the study. Treatment was started at $150 \mathrm{mg} /$ day, escalated to $300 \mathrm{mg} /$ day one week later, and to $450 \mathrm{mg} /$ day after another week. The dose was adjusted (increased or decreased) until Visit 5 of the study, after which the maintenance dose was either $300 \mathrm{mg} /$ day or $450 \mathrm{mg} /$ day.

Desensitization methods that would affect the assessment of pain associated with FM, such as non-drug therapies (for example, physical therapy, massage, chiropractry, psychotherapy, counseling) were not permitted within 30 days of the start of the screening phase through to the end of the study. Patients were permitted to use acetaminophen $(\leq 1.5 \mathrm{~g} /$ day) or nonsteroidal anti-inflammatory drugs (NSAIDs) for additional pain relief, although for 


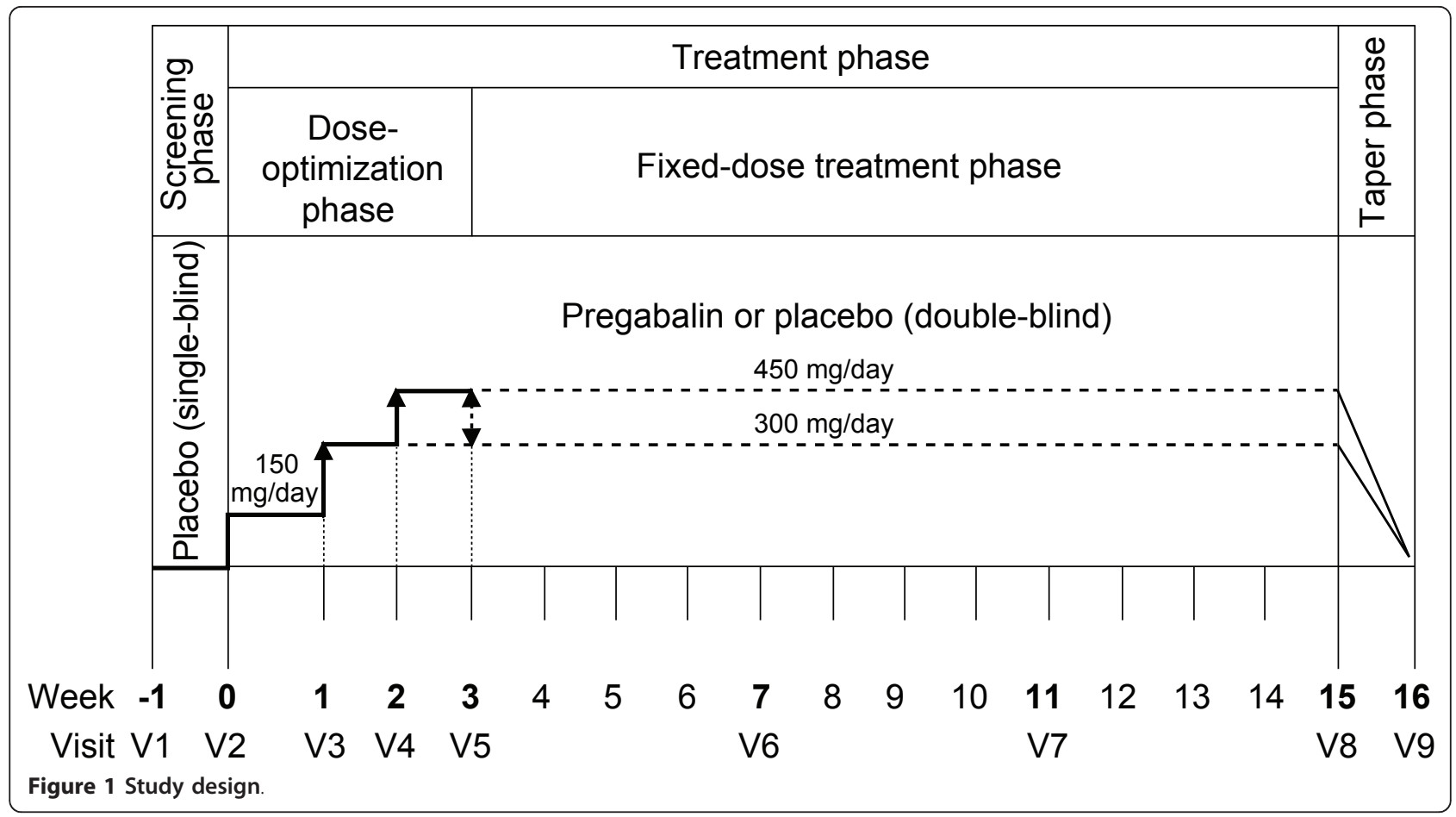

NSAIDs, the patient must have already been on a stable regimen for longer than 30 days and the dosage was not to be changed. The majority of patients had some concomitant drug use during the trial: $87.6 \%$ of patients in the pregabalin group and $88.7 \%$ of patients in the placebo group. The most common (in $\geq 10 \%$ of patients) were paracetamol (27.2\% pregabalin, $31.5 \%$ placebo), mydrin P (28.4\% pregabalin, $29.8 \%$ placebo), rebamipide $(12.8 \%$ pregabalin, $14.1 \%$ placebo) and loxoprofen sodium (10\% pregabalin, $12.1 \%$ placebo).

\section{Efficacy assessments}

The primary efficacy endpoint was the mean pain score at final assessment as recorded by patients in their daily pain diaries over the past seven days, measured using an 11 -point (0 (no pain) to 10 (worst possible pain)) numeric rating of the amount of pain over the past 24 hours $[17,18]$. The mean pain score was assessed at each week during the treatment phase until final assessment.

There were several secondary endpoints: pain VAS, with scores ranging from 0 (no pain) to $100 \mathrm{~mm}$ (worst possible pain); Patient Global Impression of Change (PGIC), a patient-rated instrument measuring the change in overall status on a scale of 1 (very much improved) to 7 (very much worse) [17]; FM Impact Questionnaire (FIQ; Japanese version), a self-administered questionnaire with 10 subscales measuring FM symptom and function domains, with the total score ranging from 0 (no impact) to 100 (maximum impact) [19,20]; SF-36 health survey measuring health-related quality of life [21]; Hospital
Anxiety and Depression Scales (HADS) measuring the presence and severity of symptoms of anxiety and depression [22]; quality of sleep score assessed by an 11-point numeric rating scale ranging from 0 (best possible sleep) to 10 (worst possible sleep) and expressed as a mean value over the past seven days [23]; Medical Outcomes Study (MOS)-Sleep Scale, a 12-item questionnaire yielding 7 subscales, each scored from 0 to 100, with higher scores indicating more of the attribute named in the subscale except for 'sleep quantity', which was scored from 0 to 24 (indicating the number of hours of sleep) and 'optimal sleep', scored as the number of patients reporting optimal sleep [24].

\section{Safety and tolerability}

Safety and tolerability were assessed by the study investigator by monitoring adverse events (AEs); the severity of each $\mathrm{AE}$ and its relationship to the study drug were recorded at each visit. Assessments included: body weight, blood pressure, pulse rate, physical examinations, edema assessment, neurological examinations and ophthalmologic examinations, 12-lead electrocardiogram, clinical laboratory testing (hematology, serum chemistry, urinalysis), and Columbia-Suicide Severity Rating Scale (C-SSRS), in addition to progression/worsening of underlying disease.

\section{Statistical analysis}

Based on results from prior studies of pregabalin in FM [10,12-14], a sample size of 498 patients was estimated to 
be sufficient to detect a clinically significant difference (at the level of 0.025 for a one-sided test) in the primary endpoint.

The primary analysis was a comparison between the pregabalin and placebo groups at final assessment and was based on an analysis of covariance model, including dose groups and baseline mean pain score as factors. The mean pain score was also calculated each week in the treatment phase and analyzed as the time-course measurement data. A mixed-effect model taking baseline value as covariate was used for the analysis, which included patients as the random effect and dose groups, points at time of evaluation, and interaction between a dose group and its point at time of evaluation as the fixed effects. A one-sided test with a significance level of 0.025 was used for analyses other than mean pain score responders (patients with a $\geq 30 \%$ or $\geq 50 \%$ reduction in their mean pain score at final assessment) and PGIC, which used a two-sided test with significance level of 0.05 . All final assessment measures were determined using last observation carried forward (LOCF).

Efficacy analyses were performed for all patients who had received $\geq 1$ dose of treatment and had $\geq 1$ entry in their daily pain diary while on study medication. Safety analyses were performed for all patients who had received $\geq 1$ dose of treatment.

\section{Results}

\section{Patient population}

A total of 501 patients were randomized (251 to pregabalin and 250 to placebo), of whom 498 received $\geq 1$ dose of study drug (250 pregabalin and 248 placebo); 415 completed the trial (Figure 2). Of the 250 patients receiving $\geq 1$ dose of pregabalin treatment, 59 received $300 \mathrm{mg} /$ day from the start of the fixed-dose period (Week 3), while 178 received $450 \mathrm{mg} /$ day (13 patients discontinued prior to Week 3 of the study). The overall study population was predominantly female with 443 women (89\%) and 55 men (11\%). The mean age was 47.9 years in the pregabalin group and 46.7 years in the placebo group. Demographic characteristics of patients assigned to each treatment arm were comparable (Table 1). The mean (range) number of months since FM onset was $62.0(0.3,508.8)$ in the placebo group and $69.6(0.3,505.1)$ in the pregabalin group. Overall, 83 patients withdrew from the study (40 in the placebo group and 43 in the pregabalin group), with 19 withdrawing from the pregabalin group due to an $\mathrm{AE}$ related to the study drug.

\section{Pain responses}

Mean pain score at final assessment was reduced from baseline with both pregabalin (a reduction of 1.48 to 5.01 final score) and placebo (a reduction of 1.03 to 5.45). The difference in reduction in pain score with pregabalin treatment compared with placebo was statistically significant $(-0.44 ; 95 \%$ CI $(-0.78,-0.11) ; P=0.0046)$. Baseline mean ( \pm standard deviation) pain scores were similar for the pregabalin $(6.5 \pm 1.3)$ and placebo groups $(6.4 \pm 1.3)$. Pregabalin treatment also resulted in a statistically significant improvement in the weekly least squares (LS) mean pain score at every time point compared with placebo (Figure 3).

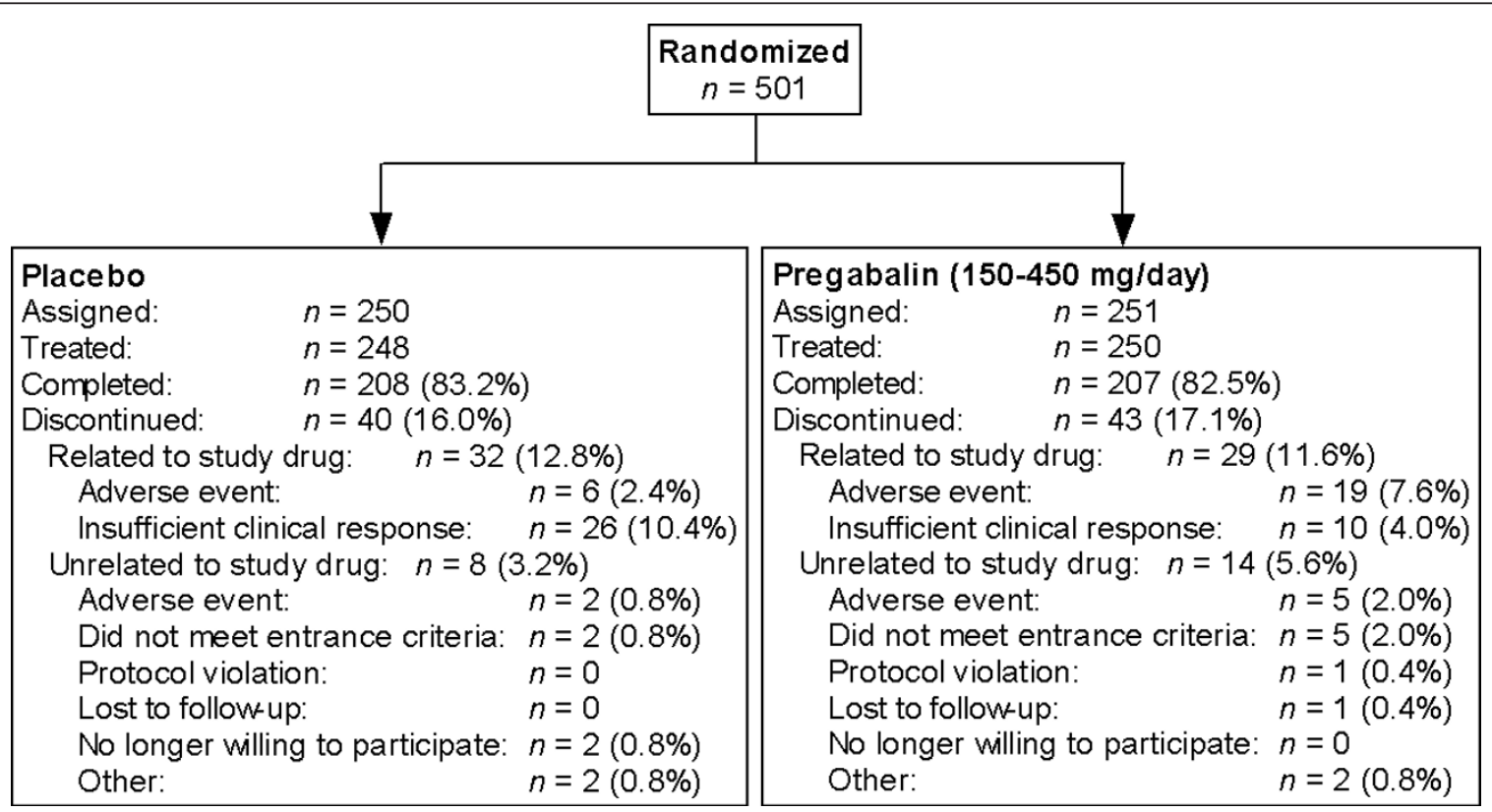

Figure 2 Patient disposition. 
Table 1 Patient characteristics

\begin{tabular}{|c|c|c|}
\hline & $\begin{array}{l}\text { Placebo } \\
N=248\end{array}$ & $\begin{array}{c}\text { Pregabalin } \\
N=250\end{array}$ \\
\hline \multicolumn{3}{|l|}{ Sex, $n(\%)$} \\
\hline Male & $31(12.5)$ & $24(9.6)$ \\
\hline Female & $217(87.5)$ & $226(90.4)$ \\
\hline \multicolumn{3}{|l|}{ Hormonal status, $n(\%)$} \\
\hline Premenopausal & $133(61.3)$ & $140(61.9)$ \\
\hline Postmenopausal & $84(38.7)$ & $86(38.1)$ \\
\hline \multicolumn{3}{|l|}{ Age (years) } \\
\hline$<18, n(\%)$ & 0 & 0 \\
\hline 18 to $44, n(\%)$ & $104(41.9)$ & $101(40.4)$ \\
\hline 45 to $64, n(\%)$ & $123(49.6)$ & $125(50.0)$ \\
\hline$\geq 65, n(\%)$ & $21(8.5)$ & $24(9.6)$ \\
\hline Mean \pm SD & $46.7 \pm 12.6$ & $47.9 \pm 12.0$ \\
\hline Range & 19,78 & 19,80 \\
\hline \multicolumn{3}{|l|}{ Weight, kg } \\
\hline Mean \pm SD & $56.2 \pm 9.0$ & $55.5 \pm 11.0$ \\
\hline Range & $38.9,90.9$ & $37.2,104.8$ \\
\hline \multicolumn{3}{|l|}{ Height, cm } \\
\hline Mean \pm SD & $158.5 \pm 6.8$ & $158.3 \pm 6.4$ \\
\hline Range & $131.9,179.6$ & $143.8,176.0$ \\
\hline \multicolumn{3}{|c|}{ Duration since FM onset, months } \\
\hline Mean & 62.0 & 69.6 \\
\hline Range & $0.3,508.8$ & $0.3,505.1$ \\
\hline
\end{tabular}

FM, fibromyalgia; SD, standard deviation

Patients with a $\geq 50 \%$ reduction in their mean pain score at final assessment ( $\geq 50 \%$ responders) accounted for $22.8 \%$ ( 57 of 250 ) of the pregabalin group compared with $12.1 \%$ (30 of 248) of the placebo group; this difference was statistically significant $(P=0.0017)$. This represents a number needed to treat (NNT) of 10. Patients with a $\geq 30 \%$ reduction in their mean pain score at final

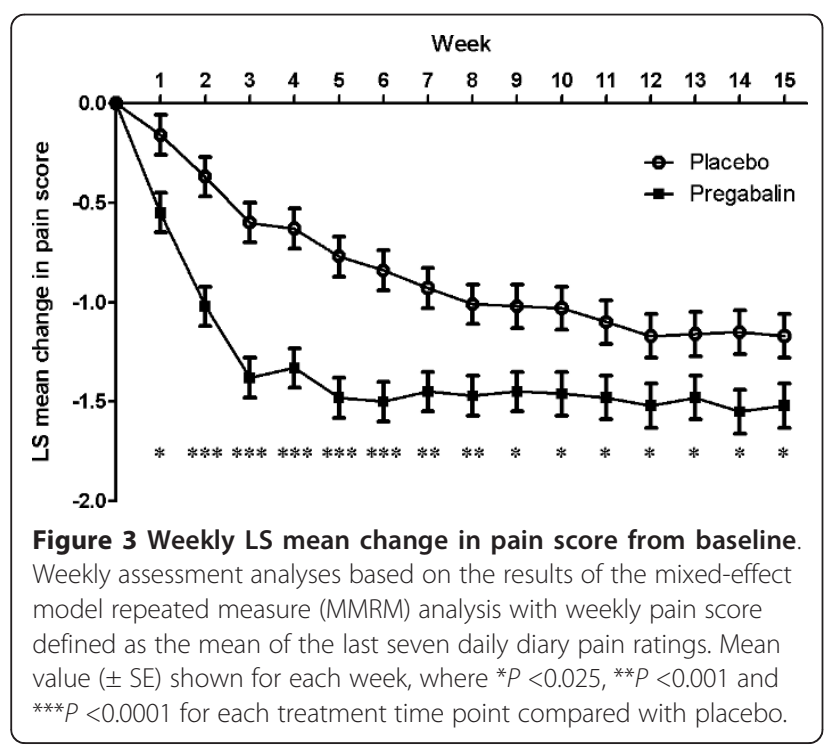

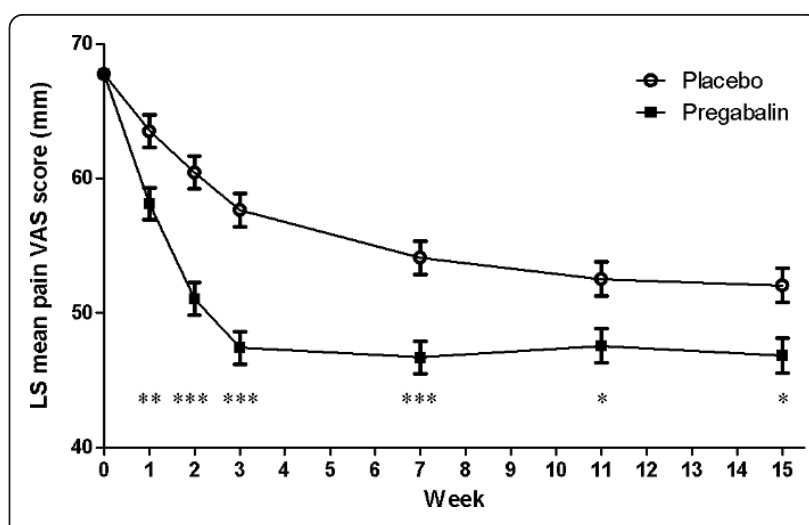

Figure 4 LS mean pain VAS score. Weekly assessment analyses based on the results of the MMRM analysis with weekly mean VAS score. Scores range from 0 to 100 with higher scores indicating increased pain. Mean value ( \pm SE) shown for each time point, where ${ }^{*} P \leq 0.025,{ }^{* *} P \leq 0.001$ and ${ }^{* * *} P \leq 0.0001$ for each treatment time point compared with placebo.

assessment ( $\geq 30 \%$ responders) accounted for $40.4 \%$ (101 of 250 ) of the pregabalin group compared with $30.6 \%$ (76 of 248) of the placebo group; this difference was also statistically significant $(P=0.0230)$.

Patients treated with pregabalin demonstrated a statistically significant improvement in pain VAS score at final assessment when compared with the placebo group (LS mean score at final assessment, 47.42 (SE, 1.44); difference from placebo -6.19 ; $95 \%$ CI $(-10.20,-2.18) ; P=$ $0.0013)$. In addition, there was a statistically significant improvement at each time point at which pain VAS was assessed from Week 1 to Week 15 (Figure 4). Baseline mean ( \pm standard deviation) pain VAS scores were similar for the pregabalin $(68.3 \pm 13.1)$ and placebo groups $(67.2 \pm 13.1)$.

\section{Patient Global Impression of Change}

The proportion of patients who reported a notable improvement in their PGIC (either "very much improved" or "much improved" [17]) was higher in the pregabalin group (38.6\%; 96 of 249 patients) than in the placebo group (26.7\%; 66 of 247 patients). Overall, patients treated with pregabalin demonstrated a statistically significantly greater improvement compared with the placebo group $(P=0.0078)$ (Table 2$)$.

\section{Fibromyalgia Impact Questionnaire}

There was a significantly greater improvement in FIQ total score in the pregabalin group (LS mean change from baseline, -10.59) compared with the placebo group $(-7.26)$ (difference from placebo, -3.33 ; 95\% CI (-6.31, -0.35); $P=0.0144$ ) (Table 3). The subscales of feeling good, pain, fatigue and morning tiredness were all significantly improved with pregabalin treatment compared 
Table 2 Patient Global Impression of Change at final assessment

\begin{tabular}{|c|c|c|}
\hline & Placebo & Pregabalin \\
\hline Number assessed ${ }^{a}$ & 247 & 249 \\
\hline Very much improved & $16(6.5)^{b}$ & $31(12.4)$ \\
\hline Much improved & $50(20.2)$ & $65(26.1)$ \\
\hline Minimally improved & $88(35.6)$ & $79(31.7)$ \\
\hline No change & $60(24.3)$ & $43(17.3)$ \\
\hline Minimally worse & $14(5.7)$ & $14(5.6)$ \\
\hline Much worse & $13(5.3)$ & $13(5.2)$ \\
\hline Very much worse & $6(2.4)$ & $4(1.6)$ \\
\hline$P$-value ${ }^{c}$ & \multicolumn{2}{|c|}{0.0078} \\
\hline
\end{tabular}

${ }^{a}$ Number of patients with available data for this analysis. ${ }^{b}$ Number (\%) of patients. ${ }^{\mathrm{C}}$ Based on chi-square test with a modified rigid transformation.

with placebo, whereas the subscales of physical functioning, housework, anxiety and stiffness showed a numerical improvement that was not statistically significant.

\section{Health-related quality of life}

The SF-36 survey revealed statistically significant improvements in physical functioning $(P=0.0006)$ and vitality $(P=0.0052)$ in the pregabalin group compared with the placebo group (Table 3). There was a numerical, but not statistically significant, trend towards improvement in the pregabalin group compared with the placebo group for mental health, bodily pain, general health perception, physical role limitations and social functioning. However, there was no significant difference between the groups for the assessment of emotional role limitations.

\section{Anxiety and depression}

Mean baseline scores ( \pm standard deviation) for the anxiety and depression subscales of the HADS were similar for the pregabalin (anxiety, $6.0 \pm 4.1$; depression, $6.1 \pm 4.2$ ) and placebo groups (anxiety, $5.8 \pm 3.7$; depression, $5.9 \pm 3.8$ ). Although there was a trend towards improvement in the anxiety subscale in the pregabalin group (LS mean score at endpoint, 5.29) compared with the placebo group (5.77), the difference was not statistically significant (LS mean change, difference from placebo -0.48 ; $95 \%$ CI $(-0.97,0.01) ; P=$ 0.0262). Similarly, there was a trend towards improvement in the depression subscale in the pregabalin group (LS mean score at endpoint, 5.71) compared with the placebo group (5.99), which was not significant (LS mean change, difference from placebo $-0.28 ;(-0.83$, 0.27); $P=0.1561$ ).

Table 3 Fibromyalgia Impact Questionnaire and SF-36 scores at baseline and final assessment

\begin{tabular}{|c|c|c|c|c|c|c|c|}
\hline \multirow[t]{2}{*}{ Assessment } & \multicolumn{2}{|c|}{$\begin{array}{l}\text { Baseline mean } \\
\text { score } \pm \text { SD }\end{array}$} & \multicolumn{2}{|c|}{$\begin{array}{l}\text { Final assessment LS mean } \\
\text { score } \pm \text { SE }\end{array}$} & \multicolumn{3}{|c|}{$\begin{array}{l}\text { Placebo-adjusted LS mean change from baseline } \\
\text { with pregabalin }{ }^{\mathrm{a}}\end{array}$} \\
\hline & Placebo & Pregabalin & Placebo & Pregabalin & Change & $95 \% \mathrm{Cl}$ & $P$-value \\
\hline \multicolumn{8}{|l|}{ FIQ score sc $^{b}$} \\
\hline Morning tiredness & $6.6 \pm 2.1$ & $6.8 \pm 2.2$ & $5.73 \pm 0.15$ & $5.13 \pm 0.15$ & -0.59 & $-1.01,-0.18$ & $0.0023^{*}$ \\
\hline Feeling good & $6.7 \pm 2.6$ & $6.8 \pm 2.6$ & $5.94 \pm 0.17$ & $5.30 \pm 0.17$ & -0.63 & $-1.12,-0.15$ & $0.0052^{*}$ \\
\hline Fatigue & $6.8 \pm 1.9$ & $7.0 \pm 1.9$ & $5.94 \pm 0.14$ & $5.45 \pm 0.14$ & -0.49 & $-0.89,-0.10$ & $0.0075^{*}$ \\
\hline Pain & $6.4 \pm 1.6$ & $6.5 \pm 1.6$ & $5.36 \pm 0.15$ & $4.95 \pm 0.15$ & -0.41 & $-0.81,0.00$ & $0.0238^{*}$ \\
\hline Physical functioning & $3.2 \pm 2.3$ & $3.3 \pm 2.4$ & $3.03 \pm 0.11$ & $2.74 \pm 0.11$ & -0.28 & $-0.59,0.03$ & 0.0376 \\
\hline Housework & $5.5 \pm 2.4$ & $5.7 \pm 2.5$ & $4.61 \pm 0.15$ & $4.30 \pm 0.15$ & -0.31 & $-0.74,0.11$ & 0.0729 \\
\hline Anxiety & $4.5 \pm 2.6$ & $4.6 \pm 2.6$ & $3.92 \pm 0.16$ & $3.64 \pm 0.16$ & -0.28 & $-0.72,0.15$ & 0.1011 \\
\hline Stiffness & $5.9 \pm 2.5$ & $6.0 \pm 2.5$ & $5.05 \pm 0.15$ & $4.90 \pm 0.15$ & -0.14 & $-0.57,0.29$ & 0.2568 \\
\hline Depression & $3.9 \pm 2.7$ & $3.9 \pm 2.6$ & $3.38 \pm 0.14$ & $3.34 \pm 0.14$ & -0.04 & $-0.44,0.35$ & 0.4165 \\
\hline Missing work & $2.2 \pm 3.0$ & $2.2 \pm 3.1$ & $1.89 \pm 0.15$ & $1.87 \pm 0.15$ & -0.01 & $-0.42,0.40$ & 0.4768 \\
\hline Total FIQ score & $51.6 \pm 15.0$ & $52.7 \pm 15.3$ & $44.89 \pm 1.08$ & $41.56 \pm 1.07$ & -3.33 & $-6.31,-0.35$ & $0.0144^{*}$ \\
\hline \multicolumn{8}{|l|}{ SF-36 health survey ${ }^{c}$} \\
\hline Physical functioning & $64.0 \pm 20.9$ & $63.4 \pm 20.8$ & $68.44 \pm 0.93$ & $72.73 \pm 0.93$ & 4.29 & $1.70,6.88$ & $0.0006^{*}$ \\
\hline Vitality & $37.6 \pm 19.4$ & $36.2 \pm 20.5$ & $42.01 \pm 1.22$ & $46.43 \pm 1.21$ & 4.42 & $1.04,7.80$ & $0.0052^{*}$ \\
\hline Mental health & $62.1 \pm 18.0$ & $60.1 \pm 20.3$ & $64.47 \pm 0.98$ & $67.11 \pm 0.98$ & 2.64 & $-0.08,5.37$ & $0.0287^{*}$ \\
\hline Bodily pain & $34.6 \pm 14.1$ & $33.0 \pm 14.0$ & $43.27 \pm 1.06$ & $45.42 \pm 1.06$ & 2.15 & $-0.81,5.10$ & 0.0770 \\
\hline General health perception & $42.6 \pm 15.7$ & $41.4 \pm 16.0$ & $44.82 \pm 0.85$ & $46.65 \pm 0.85$ & 1.83 & $-0.54,4.19$ & 0.0648 \\
\hline Physical role limitations & $52.3 \pm 26.1$ & $52.7 \pm 27.9$ & $60.90 \pm 1.30$ & $62.58 \pm 1.30$ & 1.68 & $-1.93,5.29$ & 0.1805 \\
\hline Social functioning & $61.4 \pm 26.0$ & $61.9 \pm 28.2$ & $68.51 \pm 1.38$ & $70.10 \pm 1.37$ & 1.59 & $-2.23,5.41$ & 0.2068 \\
\hline Emotional role limitations & $71.7 \pm 26.6$ & $67.2 \pm 27.2$ & $72.99 \pm 1.36$ & $72.76 \pm 1.35$ & -0.23 & $-4.00,3.54$ & 0.5480 \\
\hline
\end{tabular}

${ }^{a}$ Difference in mean change from baseline compared with placebo, using the baseline score as covariate. ${ }^{b} \mathrm{FIQ}$ scores for each assessment range from 0 to 10 with higher scores indicating greater impairment (total score range is from 0 to 100 ). ' $S F-36$ health survey scores range from 0 to 100 , with higher scores indicating better patient status. *Indicates statistical significance at the $P<0.025$ level. Cl, confidence interval; FIQ, Fibromylagia Impact Questionnaire; LS, least squares; SD, standard deviation; $\mathrm{SE}$, standard error. 


\section{Quality of sleep}

Baseline mean ( \pm standard deviation) sleep quality scores were similar for the pregabalin $(5.8 \pm 1.7)$ and placebo (5.6 \pm 1.7$)$ groups. The LS mean change in quality of sleep score was significantly better in the pregabalin group (as shown by a reduction of 1.52 to 4.17) compared with the placebo group (reduction of 0.79 to 4.91 ) at final assessment (pregabalin difference from placebo, -0.73 ; 95\% CI $(1.06,-0.40) ; P<0.0001)$, and at every time point from Week 1 through Week $15(P \leq 0.0001)$ (Figure 5$)$.

Patients treated with pregabalin showed significant improvement in the following MOS-Sleep Scale questionnaire items: sleep disturbance $(P<0.0001)$, sleep adequacy $(P<0.0001)$, quantity of sleep $(P=0.0007)$, awakening short of breath or with headache $(P=0.0049)$ and the composite overall sleep problems index $(P=0.0137)$, compared with placebo (Table 4). There was a trend towards an increase in the proportion of patients reporting optimal sleep in the pregabalin group (28.5\%; 71 of 249 patients) compared with the placebo group (21.5\%; 53 of 247 patients), but this was not statistically significant $(P=$ 0.0687). Scores on the snoring and somnolence subscales were increased (worsened) with pregabalin compared with placebo (Table 4).

\section{Safety and tolerability}

The incidence of all-causality AEs was higher in the pregabalin group (occurring in 225 of 250 patients, 90.0\%) than in the placebo group (175 of 248 patients, $70.6 \%$ ) (Table 5). Similarly, the incidence of treatment-related AEs was higher in the pregabalin group (206 of 250 patients, $82.4 \%$ ) than in the placebo group (128 of 248 patients, $51.6 \%$ ). The most common AEs in this study were somnolence, dizziness, nasopharyngitis, increased

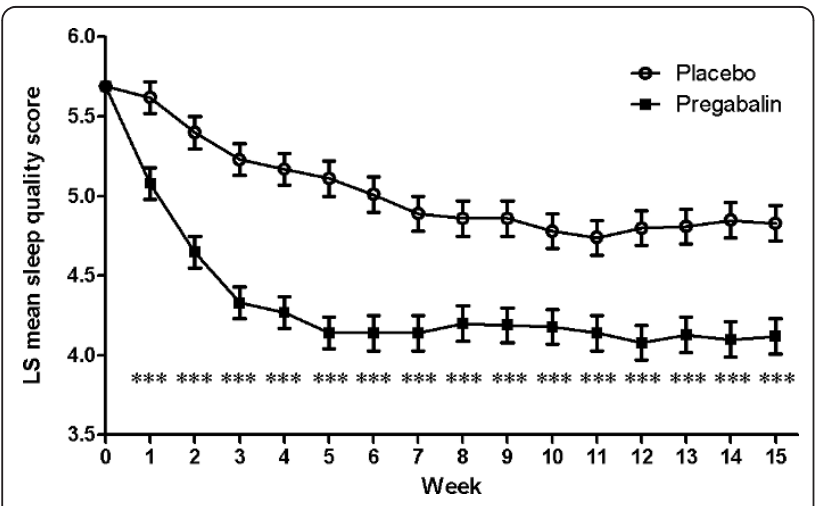

Figure 5 Weekly LS mean sleep quality score. Weekly assessment analyses based on the results of the MMRM analysis with weekly mean sleep quality score defined as the mean of the last seven daily diary sleep quality ratings. Scores range from 0 to 10 with higher scores indicating decreased sleep quality. Mean value $\left( \pm\right.$ SE) shown for each week, where ${ }^{* *} P \leq 0.0001$ for each treatment time point compared with placebo. weight and constipation with pregabalin treatment, and somnolence and nasopharyngitis with placebo (Table 5). A greater proportion of patients in the pregabalin group than in the placebo group withdrew from the study because of all-cause AEs (9.6\% vs 3.6\%, respectively). This represents a number needed to harm (NNH) of 16 . The all-cause AEs resulting in withdrawal from the study in more than one patient in the pregabalin group were somnolence (eight patients), dizziness (five patients) and insomnia (two patients).

A laboratory test result of increased creatine kinase was more frequent in the pregabalin group (7 of 250 patients, $2.8 \%$ ) than in the placebo group (1 of 248 patients, $0.4 \%$ ), although all cases were of mild severity. Increased weight was reported more frequently in the pregabalin group (39 of 250 patients, $15.6 \%$ (38 mild, 1 moderate) ) than in the placebo group ( 9 of 248 patients, $3.6 \%$ ( 8 mild, 1 moderate)). There were no clinically significant changes in blood pressure or pulse rate in the pregabalin group.

There were four serious AEs in four patients (0.8\%) who had received $\geq 1$ dose of treatment; one patient was from the placebo group (abnormal liver function test result) and three were from the pregabalin group (breast cancer, viral gastroenteritis and musculoskeletal stiffness). Severe AEs were observed in two patients from the pregabalin group (breast cancer and loss of consciousness, each in one patient) and all other AEs were mild or moderate. As assessed by the study investigators, there was no causal relationship to the study drug for any serious or severe AEs.

Suicidal ideation (mild), as rated by C-SSRS, was noted in two patients in the pregabalin group. The investigator (a physician specializing in psychosomatic medicine) had noted suicidal ideation in each of these patients prior to the start of the study. In each case, incidence was attributable to family environment and was judged to have no causal relationship to the study drug. The suicidal ideation in these cases was not judged by the study investigator to be a real desire, and treatment with the study drug was continued.

\section{Discussion}

This randomized controlled trial demonstrated the efficacy and safety of pregabalin ( 300 or $450 \mathrm{mg} /$ day) for the treatment of Japanese patients with FM. Improvements in mean pain score and pain VAS score were observed within one week and continued for the duration of the study. Treatment also significantly improved physical functioning, health-related quality of life and subjective measures of sleep.

Previous randomized, double-blind, placebo-controlled trials conducted in the US demonstrated the efficacy of pregabalin (300, 450 and $600 \mathrm{mg} /$ day) on pain, PGIC and 
Table 4 MOS-Sleep Scale scores at baseline and final assessment

\begin{tabular}{|c|c|c|c|c|c|c|c|}
\hline \multirow[t]{2}{*}{ Assessment } & \multicolumn{2}{|c|}{$\begin{array}{l}\text { Baseline mean } \\
\text { score } \pm \text { SD }\end{array}$} & \multicolumn{2}{|c|}{$\begin{array}{l}\text { Final assessment LS } \\
\text { mean score } \pm \mathrm{SE}\end{array}$} & \multicolumn{3}{|c|}{$\begin{array}{c}\text { Placebo-adjusted LS mean change from } \\
\text { baseline with pregabalin }\end{array}$} \\
\hline & Placebo & Pregabalin & Placebo & Pregabalin & Change & $95 \% \mathrm{Cl}$ & $P$-value \\
\hline Sleep disturbance ${ }^{b}$ & $47.7 \pm 26.1$ & $48.0 \pm 26.5$ & $39.76 \pm 1.31$ & $30.27 \pm 1.31$ & -9.48 & $-13.12,-5.85$ & $<0.0001^{*}$ \\
\hline Sleep adequacy & $29.0 \pm 23.7$ & $28.7 \pm 24.5$ & $36.91 \pm 1.41$ & $44.39 \pm 1.40$ & 7.48 & $3.58,11.38$ & $<0.0001^{*}$ \\
\hline Quantity of sleep & $5.4 \pm 1.4$ & $5.6 \pm 1.3$ & $5.70 \pm 0.06$ & $5.99 \pm 0.06$ & 0.29 & $0.11,0.47$ & $0.0007^{*}$ \\
\hline Awakening short of breath/headache & $25.8 \pm 26.6$ & $26.2 \pm 26.1$ & $22.99 \pm 1.36$ & $18.00 \pm 1.36$ & -4.99 & $-8.77,-1.21$ & $0.0049^{*}$ \\
\hline Snoring & $26.5 \pm 28.7$ & $25.0 \pm 28.1$ & $24.19 \pm 1.33$ & $29.17 \pm 1.33$ & 4.98 & $1.29,8.68$ & 0.9958 \\
\hline Somnolence & $41.6 \pm 23.3$ & $40.5 \pm 24.3$ & $36.41 \pm 1.29$ & $47.71 \pm 1.28$ & 11.31 & $7.74,14.87$ & 1.0000 \\
\hline Overall sleep problems index & $49.8 \pm 17.2$ & $49.7 \pm 18.5$ & $42.66 \pm 0.96$ & $39.67 \pm 0.95$ & -2.99 & $-5.65,-0.33$ & $0.0137^{*}$ \\
\hline
\end{tabular}

${ }^{a}$ Difference in mean change from baseline, compared with placebo using the baseline score as covariate. ${ }^{b}$ MOS-Sleep Scale subscales scored from 0 to 100 with higher scores indicating more of the attribute named in the subscale except for 'quantity of sleep', scored from 0 to 24 indicating the number of hours of sleep. *Indicates statistical significance at the $\mathrm{P}<0.025$ level.

$\mathrm{Cl}$, confidence interval; LS, least squares; MOS, Medical Outcomes Study; SD, standard deviation; SE, standard error

sleep over 8 [12], 13 [13] and 14 [10] weeks in FM patients. Collectively, the results from these trials supported the approval of pregabalin for the treatment of FM by the US Food and Drug Administration in June 2007. An international, 14-week trial was also conducted in patients with FM from 16 countries (Australia, Canada, Denmark, France, Germany, India, Italy, Korea, Mexico, Portugal, Spain, Sweden, Switzerland, The Netherlands, UK and Venezuela). This international study demonstrated modest efficacy on pain, PGIC and FIQ scores with pregabalin $450 \mathrm{mg} /$ day, although data at 300 and $600 \mathrm{mg} /$ day were inconsistent [14], and the European Medicines Agency (EMA) did not approve pregabalin for the treatment of FM. Currently, there are no approved treatments for FM in the European Union.

Results from the current study in Japanese patients were broadly consistent with those from previous trials, in which reductions in mean pain score (vs placebo) ranged from -0.47 to $-0.98[10,12-14]$, compared with -0.44 in the current study. In addition, similar proportions of patients had $\mathrm{a} \geq 30 \%$ or $\geq 50 \%$ reduction in their mean pain score, with 34 to $50 \%$ achieving a $\geq 30 \%$ reduction versus 19 to $35 \%$ with placebo (compared with $40.4 \%$ vs $30.6 \%$ in this study) and 18 to $29 \%$ achieving a $\geq 50 \%$ reduction versus 9 to $15 \%$ with placebo (compared with $22.8 \%$ vs $12.1 \%$ in this study). The NNT to achieve a $\geq 50 \%$ reduction in mean pain score in these previous studies ranged from 7 to $12[10,12,14]$ (compared with 10 in this study).

While the primary objective of this trial was to assess the effect of pregabalin on the symptomatic relief of pain, the study also assessed the broader effects of pregabalin on overall health status and quality of life. Measures such as PGIC allow patients to provide their own assessment of their overall health status, taking into consideration pain and other symptoms together with physical and emotional functioning, and any adverse effects. This

Table 5 All-cause adverse events

\begin{tabular}{|c|c|c|}
\hline & $\begin{array}{l}\text { Placebo } \\
N=\mathbf{2 4 8}\end{array}$ & $\begin{array}{c}\text { Pregabalin } \\
N=250\end{array}$ \\
\hline Adverse events & $175(70.6)^{a}$ & $225(90.0)$ \\
\hline Serious adverse events & $1(0.4)$ & $3(1.2)$ \\
\hline Severe adverse events & 0 & $2(0.8)$ \\
\hline Discontinuations due to adverse events & $9(3.6)$ & $24(9.6)$ \\
\hline Dose reductions/temporary discontinuations due to adverse events & $11(4.4)$ & $30(12.0)$ \\
\hline \multicolumn{3}{|l|}{ Frequent adverse events $^{\mathrm{b}}$} \\
\hline Somnolence & $45(18.1)$ & $116(46.4)$ \\
\hline Dizziness & $15(6.0)$ & $74(29.6)$ \\
\hline Nasopharyngitis & $45(18.1)$ & $45(18.0)$ \\
\hline Increased weight & $9(3.6)$ & $39(15.6)$ \\
\hline Constipation & $17(6.9)$ & $36(14.4)$ \\
\hline Feeling abnormal & $3(1.2)$ & $20(8.0)$ \\
\hline Peripheral edema & $3(1.2)$ & $18(7.2)$ \\
\hline Headache & $15(6.0)$ & $15(6.0)$ \\
\hline Vision blurred & $3(1.2)$ & $13(5.2)$ \\
\hline
\end{tabular}

${ }^{a}$ Number (\%) of patients. ${ }^{b}$ Reported by $\geq 5 \%$ of patients in any group. 
study demonstrated a significant improvement in PGIC with pregabalin compared with placebo, with $38.6 \%$ vs $26.7 \%$ of patients reporting that their symptoms were either "very much improved" or "much improved". These results were consistent with prior trials, in which the percentage point difference between pregabalin and placebo in PGIC ranged from 6.2 to 26.0 [10,12-14]. Similarly, there was a numerical improvement with pregabalin compared with placebo in all 10 subscales of the FIQ, an assessment that quantifies the overall impact of FM on patients' functioning across the spectrum of problems associated with the disorder [25]. The improvement was statistically significant for four of the subscales: feeling good, pain, fatigue and morning tiredness. There was also a trend towards improvement with pregabalin compared with placebo in seven of the eight subscales on the SF-36, statistically significant in two (physical functioning and vitality).

Sleep disturbance is one of the key clinical domains in FM [6]. There is a distinct relationship between poor quality sleep and pain, with pain then potentially leading to decreased physical functioning and depression [26-28]. In this study, pregabalin significantly improved measures of sleep by two distinct instruments: the quality of sleep score and the MOS-Sleep Scale questionnaire. All measures on the MOS-Sleep Scale questionnaire showed improvement with pregabalin, with the exception of the somnolence and snoring subscales. This was not unexpected for somnolence, which is a common AE with pregabalin [16]. The observed improvements in subjective measures of sleep in this study were consistent with prior trials of pregabalin in both FM [10-13] and other conditions, such as restless legs syndrome [29] and various types of neuropathic pain [30-33]. Pregabalin has also been shown to significantly improve objective measures of sleep in a four-week polysomnography study measuring Wake After Sleep Onset in FM patients [34].

There were no serious or severe treatment-related AEs observed in this study, although not all patients tolerated pregabalin and discontinuations and dose reductions were more common with pregabalin than placebo. The majority of discontinuations were due to somnolence or dizziness. The $\mathrm{NNH}$, based on discontinuations from the study, was higher in this trial (16) than in previous trials of $450 \mathrm{mg}$ /day pregabalin in FM patients (in which it ranged from 9 to 11$)[10,13,14]$, potentially influenced by the somewhat flexible, lower dosing in this study. A meta-analysis of previous pregabalin trials in neuropathic pain and fibromyalgia concluded that while pregabalin has proven efficacy, many patients will have little or no benefit, or will discontinue treatment due to adverse events, particularly at higher doses, highlighting the importance of titrating the dose for each patient to minimize AEs [35]. The higher NNH and similar NNT numbers in this study, compared with prior trials of pregabalin in FM patients, indicate that the risk:benefit profile was more favorable than in prior trials in the US and internationally despite there still being a significant discontinuation rate with pregabalin in this study $(9.6 \%$ vs $3.6 \%$ with placebo).

Overall, the safety profile of pregabalin in this population was consistent with prior clinical trials; the most common treatment-related AEs with pregabalin being somnolence and dizziness. Nevertheless, further trials are needed to assess the long-term safety and tolerability of pregabalin in Japanese patients with FM. In order to address this, this trial has been extended into a 52-week, open-label study (the results of which will be reported elsewhere).

\section{Conclusions}

In this, the first clinical trial in FM patients in Japan, pregabalin demonstrated significant efficacy in pain reduction and also improved measures of sleep and functioning. The drug was generally well tolerated, and AEs were consistent with prior trials and current product labeling. These data suggest that pregabalin may be an effective treatment option for the relief of pain and sleep problems in Japanese patients with FM.

\section{Abbreviations}

ACR: American College of Rheumatology; AE: adverse event; Cl: confidence interval; C-SSRS: Columbia Suicide Severity Rating Scale; FM: fibromyalgia; FIQ: Fibromyalgia Impact Questionnaire; HADS: Hospital Anxiety and Depression Scale; LOCF: last observation carried forward; LS: least squares; MMRM: mixed-effect model repeated measure; MOS: Medical Outcomes Study; NNH: number needed to harm; NNT: number needed to treat; NSAIDs: nonsteroidal anti-inflammatory drugs; PGIC: Patient Global Impression of Change; SF-36: 36-Item Short Form Health Survey; VAS: visual analog scale.

\section{Acknowledgements}

This study was funded by Pfizer Japan, Inc. Medical writing support was provided by Joshua Fink PhD, of UBC Scientific Solutions, and funded by Pfizer, Inc.

We would like to acknowledge the contribution of the study's principal investigators: Shinichi Aoki, Hisashi Date, Tomomi Himeno, Hiroshi Inoue, Akio Iseki, Sadahiko Kameda, Hyeteok Kim, Masamiki Kimura, Tomomasa Kimura, Keizo Kobayashi, Yoshinobu Koyama, Kojiro Kumagai, Shinichi Kuwabara, Satoe Matsubayashi, Yoshifuji Matsumoto, Hiroaki Matsuno, Kenji Miki, Akiko Miyazawa, Akihito Mizutani, Yuko Morita, Yasuhiko Munakata, Masato Murakami, Shouhei Nagaoka, Shiro Nakayama, Syuji Ohno, Hiroshi Oka, Kenryo Oka, Yoshinori Okubo, Masanari Omata, Hirofumi Oosaki, Motohiro Oribe, Yasuhiro Saito, Kenmei Sakata, Yuki Sekiguchi, Kazutoshi Seto, Eishi Shirasawa, Takao Sugiyama, Kimihiro Suzuki, Masaharu Tajima, Yoshinari Takasaki, Nobuo Takubo, Toru Tsuda, Fusazo Urano, Ryoichi Yamazaki and Masao Yukioka.

\section{Author details}

${ }^{1}$ Pfizer Japan, Inc., 3-22-7, Yoyogi, Shibuya-ku, Tokyo 151-8589, Japan. ${ }^{2}$ Rheumatic Disease Center, Tokyo Medical University Hachioji Medical Center, 1163 Tatemachi Hachioji, Tokyo 193-0998, Japan. ³epartment of Psychiatry, Juntendo University School of Medicine, Juntendo University Nerima Hospital, 3-1-10 Takanodai, Nerima-ku, Tokyo 177-8521, Japan. ${ }^{4}$ Institute of Innovative Medical Science and Education, Tokyo Medical University, 6-1-1 Shinjyuku, Shinjyuku-ku, Tokyo 160-8402, Japan. 


\section{Authors' contributions}

$\mathrm{KN}, \mathrm{HOka}, \mathrm{HOhta}, \mathrm{MO}$ and MS contributed to the design of the study. HOka was a principal investigator and KN and $\mathrm{CU}$ were sub-investigators on the study. MO conducted the statistical analysis of the data. All authors had full access to the study data, contributed to the drafting and critical review of the manuscript and read and approved the final version.

\section{Competing interests}

HOhta, MO and MS are employees of Pfizer Japan, Inc. KN and HOka received a consultancy fee from Pfizer Japan, Inc. for their participation in this study. CU declares no competing interests. KN, HOka and CU were not compensated for their work on this manuscript.

Received: 17 April 2012 Revised: 24 August 2012

Accepted: 17 September 2012 Published: 12 October 2012

\section{References}

1. Wolfe F, Smythe HA, Yunus MB, Bennett RM, Bombardier C, Goldenberg DL, Tugwell P, Campbell SM, Abeles M, Clark P, et al: The American College of Rheumatology 1990 criteria for the classification of fibromyalgia. Report of the Multicenter Criteria Committee. Arthritis Rheum 1990, 33:160-172.

2. Clauw DJ, Crofford LJ: Chronic widespread pain and fibromyalgia: what we know, and what we need to know. Best Pract Res Clin Rheumatol 2003, 17:685-701.

3. Mease P: Fibromyalgia syndrome: review of clinical presentation, pathogenesis, outcome measures, and treatment. J Rheumatol Suppl 2005, 75:6-21.

4. Mease P, Arnold LM, Choy EH, Clauw DJ, Crofford LJ, Glass JM, Martin SA, Morea J, Simon L, Strand CV, Williams DA: Fibromyalgia syndrome module at OMERACT 9: domain construct. J Rheumatol 2009, 36:2318-2329.

5. Wolfe F, Clauw DJ, Fitzcharles MA, Goldenberg DL, Katz RS, Mease P, Russell AS, Russell IJ, Winfield JB, Yunus MB: The American College of Rheumatology preliminary diagnostic criteria for fibromyalgia and measurement of symptom severity. Arthritis Care Res (Hoboken) 2010, 62:600-610.

6. Mease PJ, Arnold LM, Crofford LJ, Williams DA, Russell IJ, Humphrey L, Abetz L, Martin SA: Identifying the clinical domains of fibromyalgia: contributions from clinician and patient Delphi exercises. Arthritis Rheum 2008, 59:952-960.

7. Wolfe F, Ross K, Anderson J, Russell IJ, Hebert L: The prevalence and characteristics of fibromyalgia in the general population. Arthritis Rheum 1995, 38:19-28.

8. Matsumoto M: Epidemiology of fibromyalgia. Pharma Medica 2006 24:35-39, Japanese.

9. Nishioka K: Research platform for fibromyalgia in Japan. Arthritis Res Ther 2012, 14(Suppl 1):05.

10. Arnold LM, Russell IJ, Diri EW, Duan WR, Young JP Jr, Sharma U, Martin SA, Barrett JA, Haig G: A 14-week, randomized, double-blinded, placebocontrolled monotherapy trial of pregabalin in patients with fibromyalgia. J Pain 2008, 9:792-805.

11. Crofford LJ, Mease PJ, Simpson SL, Young JP Jr, Martin SA, Haig GM, Sharma U: Fibromyalgia relapse evaluation and efficacy for durability of meaningful relief (FREEDOM): a 6-month, double-blind, placebocontrolled trial with pregabalin. Pain 2008, 136:419-431.

12. Crofford LJ, Rowbotham MC, Mease PJ, Russell IJ, Dworkin RH, Corbin AE, Young JP Jr, LaMoreaux LK, Martin SA, Sharma U: Pregabalin for the treatment of fibromyalgia syndrome: results of a randomized, doubleblind, placebo-controlled trial. Arthritis Rheum 2005, 52:1264-1273.

13. Mease PJ, Russell IJ, Arnold LM, Florian H, Young JP Jr, Martin SA, Sharma U: A randomized, double-blind, placebo-controlled, phase III trial of pregabalin in the treatment of patients with fibromyalgia. J Rheumatol 2008, 35:502-514.

14. Pauer $L$, Winkelmann $A$, Arsenault $P$, Jespersen $A$, Whelan $L$, Atkinson $G$, Leon T, Zeiher B: An international, randomized, double-blind, placebocontrolled, phase III trial of pregabalin monotherapy in treatment of patients with fibromyalgia. J Rheumatol 2011, 38:2643-2652.

15. Field MJ, Cox PJ, Stott E, Melrose H, Offord J, Su TZ, Bramwell S, Corradini L, England S, Winks J, Kinloch RA, Hendrich J, Dolphin AC, Webb T, Williams D: Identification of the alpha2-delta-1 subunit of voltagedependent calcium channels as a molecular target for pain mediating the analgesic actions of pregabalin. Proc Natl Acad Sci USA 2006, 103:17537-17542.
16. Pfizer Inc: LYRICA prescribing information. [http://labeling.pfizer.com/ ShowLabeling.aspx?id=561].

17. Dworkin RH, Turk DC, Wyrwich KW, Beaton D, Cleeland CS, Farrar JT, Haythornthwaite JA, Jensen MP, Kerns RD, Ader DN, Brandenburg N, Burke LB, Cella D, Chandler J, Cowan P, Dimitrova R, Dionne R, Hertz S, Jadad AR, Katz NP, Kehlet H, Kramer LD, Manning DC, McCormick C, McDermott MP, McQuay HJ, Patel S, Porter L, Quessy S, Rappaport BA, et al: Interpreting the clinical importance of treatment outcomes in chronic pain clinical trials: IMMPACT recommendations. J Pain 2008, 9:105-121.

18. Farrar JT, Young JP Jr, LaMoreaux L, Werth JL, Poole RM: Clinical importance of changes in chronic pain intensity measured on an 11-point numerical pain rating scale. Pain 2001, 94:149-158.

19. Burckhardt CS, Clark SR, Bennett RM: The fibromyalgia impact questionnaire: development and validation. J Rheumatol 1991, 18:728-733.

20. Osada K, Oka H, Isomura T, Nakamura I, Tominaga K, Takahashi S, Kojima A, Nishioka K: Development of the Japanese version of the Fibromyalgia Impact Questionnaire (JFIQ): psychometric assessments of reliability and validity. Int J Rheum Dis 2011, 14:74-80.

21. Ware JE Jr, Sherbourne CD: The MOS 36-item short-form health survey (SF36). I. Conceptual framework and item selection. Med Care 1992, 30:473-483.

22. Snaith RP, Zigmond AS: The Hospital Anxiety and Depression Scale Manual London: NFER Nelson; 1994.

23. Cappelleri JC, Bushmakin AG, McDermott AM, Sadosky AB, Petrie CD, Martin S: Psychometric properties of a single-item scale to assess sleep quality among individuals with fibromyalgia. Health Qual Life Outcomes 2009, 7:54.

24. Hays RD, Stewart AL: Sleep measures. In Measuring Functioning and WellBeing. Edited by: Stewart AL, Ware JE Jr. Durham, NC: Duke University Press; 1992:235-239.

25. Bennett R: The Fibromyalgia Impact Questionnaire (FIQ): a review of its development, current version, operating characteristics and uses. Clin Exp Rheumatol 2005, 23:S154-162.

26. Harding SM: Sleep in fibromyalgia patients: subjective and objective findings. Am J Med Sci 1998, 315:367-376.

27. Moldofsky H: The significance of dysfunctions of the sleeping/waking brain to the pathogenesis and treatment of fibromyalgia syndrome. Rheum Dis Clin North Am 2009, 35:275-283.

28. Bigatti SM, Hernandez AM, Cronan TA, Rand KL: Sleep disturbances in fibromyalgia syndrome: relationship to pain and depression. Arthritis Rheum 2008, 59:961-967.

29. Garcia-Borreguero D, Larrosa O, Williams AM, Albares J, Pascual M, Palacios JC, Fernandez C: Treatment of restless legs syndrome with pregabalin: a double-blind, placebo-controlled study. Neurology 2010, 74:1897-1904.

30. Dworkin RH, Corbin AE, Young JP Jr, Sharma U, LaMoreaux L, Bockbrader $H_{\text {, }}$ Garofalo EA, Poole RM: Pregabalin for the treatment of postherpetic neuralgia: a randomized, placebo-controlled trial. Neurology 2003, 60:1274-1283.

31. Siddall PJ, Cousins MJ, Otte A, Griesing T, Chambers R, Murphy TK: Pregabalin in central neuropathic pain associated with spinal cord injury: a placebo-controlled trial. Neurology 2006, 67:1792-1800.

32. Freynhagen R, Strojek K, Griesing T, Whalen E, Balkenohl M: Efficacy of pregabalin in neuropathic pain evaluated in a 12-week, randomised, double-blind, multicentre, placebo-controlled trial of flexible- and fixeddose regimens. Pain 2005, 115:254-263.

33. van Seventer R, Feister HA, Young JP Jr, Stoker M, Versavel M, Rigaudy L: Efficacy and tolerability of twice-daily pregabalin for treating pain and related sleep interference in postherpetic neuralgia: a 13-week randomized trial. Curr Med Res Opin 2006, 22:375-384.

34. Roth T, Lankford DA, Bhadra P, Whalen E, Resnick EM: Effect of pregabalin on sleep in patients with fibromyalgia and sleep maintenance disturbance: a randomized, placebo-controlled, 2-way crossover polysomnography study. Arthritis Care Res (Hoboken) 2012, 64:597-606.

35. Moore RA, Straube S, Wiffen PJ, Derry S, McQuay HJ: Pregabalin for acute and chronic pain in adults. Cochrane Database Syst Rev 2009, CD007076.

\section{doi:10.1186/ar4056}

Cite this article as: Ohta et al:: A randomized, double-blind, multicenter, placebo-controlled phase III trial to evaluate the efficacy and safety of pregabalin in Japanese patients with fibromyalgia. Arthritis Research \& Therapy 2012 14:R217. 